\title{
Two-dimensional systems with competing interactions: microphase formation under the effect of a disordered porous matrix
}

\author{
D.F. Schwanzer, G. Kahl \\ Institut für Theoretische Physik and Center for Computational Materials Science (CMS), Technische \\ Universität Wien, Wiedner Hauptstraße 8-10, A-1040 Wien, Austria
}

Received May 18, 2011, in final form July 4, 2011

\begin{abstract}
We have investigated the effect of a disordered porous matrix on the cluster microphase formation of a two dimensional system where fluid particles interact via competing interactions. To this end we have performed extensive Monte Carlo simulations and have systematically varied the densities of the fluid and of the matrix as well as the interaction between the matrix particles and between the matrix and fluid particles. Our results provide evidence that the matrix does have a distinct effect on the microphase formation of the fluid particles: as long as the matrix particles interact both among themselves as well as with the fluid particles via a simple hard sphere potential, they essentially reduce the available space, in which the fluid particles form a cluster microphase. On the other hand, if we turn on a long-range tail in the matrix-matrix and in the matrix-fluid interactions, the matrix particles become nucleation centers for the clusters formed by the fluid particles.
\end{abstract}

Key words: soft matter, porous media, microphase formation, static structure, dynamic properties

PACS: $82.70 . D d, 46.65 .+g, 47.54 . B d, 61.20 . J a$

\section{Introduction}

In recent years, systems with so-called competing interactions have attracted considerable interest in the scientific community. In such systems, the (effective) potential $\Phi(r)$ between two particles that are separated by a distance $r$ is given by a strongly repulsive core region and an adjacent tail which is characterized by interparticle interactions operating on different length scales: it has an attractive component at short distances and a repulsive component at intermediate distances, eventually tending to zero for $r \rightarrow \infty$. In the past, experimental investigations [1 [5] on such systems have been complemented by thorough theoretical studies 6 -17], most of which have focused on a two dimensional case.

The most remarkable feature of such systems is their capability of self-organizing at sufficiently low temperatures - despite a spherically symmetric potential - into so-called microphases, i.e., highly inhomogeneous, ordered patterns. In two dimensions, on which the present contribution will focus, as the density increases, the following morphologies are reported: clusters, stripes and bubbles (or inverse clusters). The aforementioned theoretical investigations devoted to the two dimensional case [6 - 13, 17] have also unveiled other interesting and surprising features of these systems, related to the phase behaviour and to the properties of their disordered phases. However, since these aspects are of less relevance to the present contribution we refer the reader to these references for more details.

In the present contribution we investigate the following scenario: we consider a disordered matrix, generated by a frozen (quenched) equilibrium configuration of matrix particles and immerse fluid particles (annealed component) into this configurations that interact via potentials operating on different length scales. Using Monte Carlo (MC) simulations we study the effect of the matrix on the microphase formation scenario of the fluid particles, varying the fluid and the matrix densities as well as the fluid-matrix and the matrix-matrix interactions. In these investigations we focus on a region in phase space, where the equilibrated fluid forms clusters, i.e., we concentrate on sufficiently 
low temperatures and on rather low fluid densities, $\rho_{\mathrm{f}}$. In order to generate such systems we have used the quenched-annealed concept (see, e.g., [18, 19] and references therein): in a first step, we have generated an equilibrium configuration of the matrix particles in a MC simulations at a desired matrix density $\rho_{\mathrm{m}}$. Keeping the positions of the matrix particles fixed we have then immersed a fluid of a density $\rho_{\mathrm{f}}$ into the system. Using extensive MC simulations we have evaluated, via suitable averaging processes (see, e.g., [18, 19]), the information on the static structure of the fluid and on the mobility of the fluid particles. This information provides us, in combination with a visual inspection of the snapshots, with information on the microphase formation of the fluid particles under the external field of the matrix particles.

We summarize our observations as follows: both the densities $\rho_{\mathrm{f}}$ and $\rho_{\mathrm{m}}$ as well as the type of interaction between fluid-matrix and matrix-matrix particles have a distinct effect on the observed microphase formation scenarios of the fluid particles: at small matrix densities, the cluster formation is essentially unaffected by the presence of the matrix particles. However, as $\rho_{\mathrm{m}}$ increases, the interparticle potentials both between the fluid and the matrix as well as between the matrix particles becomes relevant: (i) hard sphere matrix particles can foster or suppress microphase formation, depending on whether they leave sufficient space for the fluid particles to self-assemble in clusters or not; (ii) on the other hand, if the fluid-matrix interaction is essentially of the same type as the fluid-fluid interaction, then we observe that the matrix particles act as nucleation sites for the clusters formed by the fluid particles.

The paper is organized as follows: in the subsequent section we present our model system and summarize the details concerning the MC simulations. In section 3 we discuss our results and close the paper with concluding remarks.

\section{Model and theoretical approach}

As in our previous investigation [17], the fluid particles (index ' $f$ ') interact via a spherically symmetric potential $\Phi_{\mathrm{ff}}(r)=\Phi_{\mathrm{IR}}(r)$, originally proposed in this parametrization by Imperio and Reatto (IR) [6]; it is given by

$$
\Phi_{\mathrm{IR}}(r)= \begin{cases}\infty, & r \leqslant \sigma, \\ -\epsilon_{\mathrm{a}} \frac{\sigma^{2}}{R_{\mathrm{a}}^{2}} \exp \left(-\frac{r}{R_{\mathrm{a}}}\right)+\epsilon_{\mathrm{r}} \frac{\sigma^{2}}{R_{\mathrm{r}}^{2}} \exp \left(-\frac{r}{R_{\mathrm{r}}}\right), & r>\sigma .\end{cases}
$$

$\sigma$ is the diameter of the impenetrable hard-core region, $\epsilon_{\mathrm{a}}\left(\epsilon_{\mathrm{r}}\right)$ and $R_{\mathrm{a}}\left(R_{\mathrm{r}}\right)$ represent the strength and the range of the attractive (repulsive) contributions to the potential tail of $\Phi_{\mathrm{IR}}(r)$ for $r \geqslant \sigma$, respectively. Throughout we have used $\epsilon_{\mathrm{a}}=\epsilon_{\mathrm{r}}$ and $R_{\mathrm{a}}=\sigma$ and $R_{\mathrm{r}}=2 \sigma$. Further we introduce the temperature $T$ and the fluid and matrix (area) densities $\rho_{\mathrm{f}}$ and $\rho_{\mathrm{m}}$, respectively. Further we will use reduced units, i.e., $r^{*}=r / \sigma, k^{*}=k \sigma$, and $\rho^{*}=\rho \sigma^{2}$; for the temperature $T$ we have used the arbitrary temperature scale introduced in [6], namely that for $R_{\mathrm{r}} / R_{\mathrm{a}}=2$ and $\epsilon_{\mathrm{r}} / \epsilon_{\mathrm{a}}=1$, $\Phi(\sigma) / k_{\mathrm{B}} T=-1$. For simplicity we will drop henceforward the asterisk.

For the interactions between the matrix particles (index 'm'), $\Phi_{\mathrm{mm}}(r)$, and the cross interaction between fluid and matrix particles, $\Phi_{\mathrm{fm}}(r)$, we have used either a simple hard (HS) sphere potential, $\Phi_{\mathrm{HS}}(r)$ (with a HS diameter $\sigma$ ) or the IR potential, specified in equation (2.1). In this way we are able to study the effect of the matrix on the microphase separation scenario of the fluid particles, including thereby both simple excluded volume effects [i.e., when $\Phi_{\mathrm{fm}}(r)=\Phi_{\mathrm{HS}}(r)$ ] and energetic effects [i.e., when $\Phi_{\mathrm{fm}}(r)=\Phi_{\mathrm{IR}}(r)$ ]. To be more specific, the fluid particles always interact via $\Phi_{\mathrm{IR}}(r)$; for the fluid-matrix and the matrix-matrix interactions we have considered the following three combinations (cases 1 to 3$)$ :

- case 1: $\Phi_{\mathrm{fm}}(r)=\Phi_{\mathrm{HS}}(r)$ and $\Phi_{\mathrm{mm}}(r)=\Phi_{\mathrm{HS}}(r)$;

- case 2: $\Phi_{\mathrm{fm}}(r)=\Phi_{\mathrm{IR}}(r)$ and $\Phi_{\mathrm{mm}}(r)=\Phi_{\mathrm{HS}}(r)$;

- case 3: $\Phi_{\mathrm{fm}}(r)=\Phi_{\mathrm{IR}}(r)$ and $\Phi_{\mathrm{mm}}(r)=\Phi_{\mathrm{IR}}(r)$. 
The range of the densities $\rho_{\mathrm{f}}$ and $\rho_{\mathrm{m}}$ has been restricted to smaller values below 0.2 and, throughout, temperature was set to $T=0.5$, corresponding to the region in phase space where cluster formation is expected to occur for the pure fluid (cf. figure 4 in [17]). In figure 1 we have specified in the $\left(\rho_{\mathrm{f}}, \rho_{\mathrm{m}}\right)$-plane those systems that we have investigated in this contribution: for state points located along the path $A, \rho_{\mathrm{f}}$ is kept fixed to a value of 0.2 , while $\rho_{\mathrm{m}}$ varies from 0 up to 0.1 ; along the path $B$, the total density, $\rho_{\mathrm{t}}=\rho_{\mathrm{f}}+\rho_{\mathrm{m}}$, is kept fixed to a value of 0.2 .

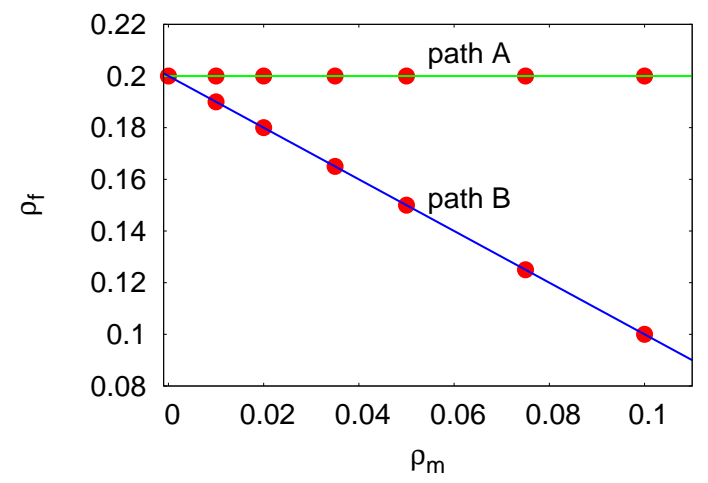

Figure 1. (color online) Symbols in the $\left(\rho_{\mathrm{f}}, \rho_{\mathrm{m}}\right)$-plane represent state points that have been investigated in this contribution. Along path $A$, the fluid density is fixed to $\rho_{\mathrm{f}}=0.2$, while the matrix density $\rho_{\mathrm{m}}$ varies from 0 to 0.1 . Along path $B$, the total density, $\rho_{\mathrm{t}}=\rho_{\mathrm{f}}+\rho_{\mathrm{m}}$, is kept fixed to $\rho_{\mathrm{t}}=0.2: \rho_{\mathrm{m}}$ increases from 0 to 0.1 , i.e. $\rho_{\mathrm{f}}$ decreases from 0.2 to 0.1 .

System properties have been investigated via standard NVT MC simulations. $N_{\mathrm{f}}$ fluid and $N_{\mathrm{m}}$ matrix particles have been considered in a square box, using periodic boundary conditions. Depending on the total density, from 2000 to 6000 particles have been considered. Computational speed-up was achieved by refined techniques described more in detail in subsection 2.3 of [17]. The potential (2.1) was truncated at $r_{\text {cut }}=17.2$, for the maximum displacement we used throughout a value of 0.6. Matrix configurations were created in simulation runs of an equilibrated system of matrix particles at a density $\rho_{\mathrm{m}}$. At several instances, the simulation has been halted and the positions of the particles were recorded; thereby different but equivalent matrix configurations were produced. Simulations have been extended over 1000000 MC sweeps. For a given state point, characterized by $\rho_{\mathrm{f}}$ and $\rho_{\mathrm{m}}$, observables were obtained in a two-step averaging procedure: in a first step, an average was taken over the degrees of freedom of the fluid particles for a given matrix configuration (involving 10000 independent particle configurations); in a second step, these results were averaged over five independent matrix configurations.

\section{Results}

We discuss the results obtained for our system along the following lines: after a qualitative visual inspection of the selected representative snapshots obtained from MC simulations, we will analyse these findings on a more quantitative level by studying the pair structure and the mean square displacement.

\subsection{Snapshots}

In figure 2 we show selected representative snapshots for systems located in the $\left(\rho_{\mathrm{f}}, \rho_{\mathrm{m}}\right)$-plane along path $A$, specified in figure 1, i.e. starting with the pure fluid of density $\rho_{\mathrm{f}}=0.2$ (i.e., $\rho_{\mathrm{m}}=0$ ) and - while keeping $\rho_{\mathrm{f}}$ fixed - increasing continuously the matrix density $\rho_{\mathrm{m}}$ from 0 to 0.1 . We recall that - according to the phase diagram depicted in figure 4 of [17] - for this density-range and for the assumed temperature, the matrix particles do not form clusters. In the top panel of figure 2 


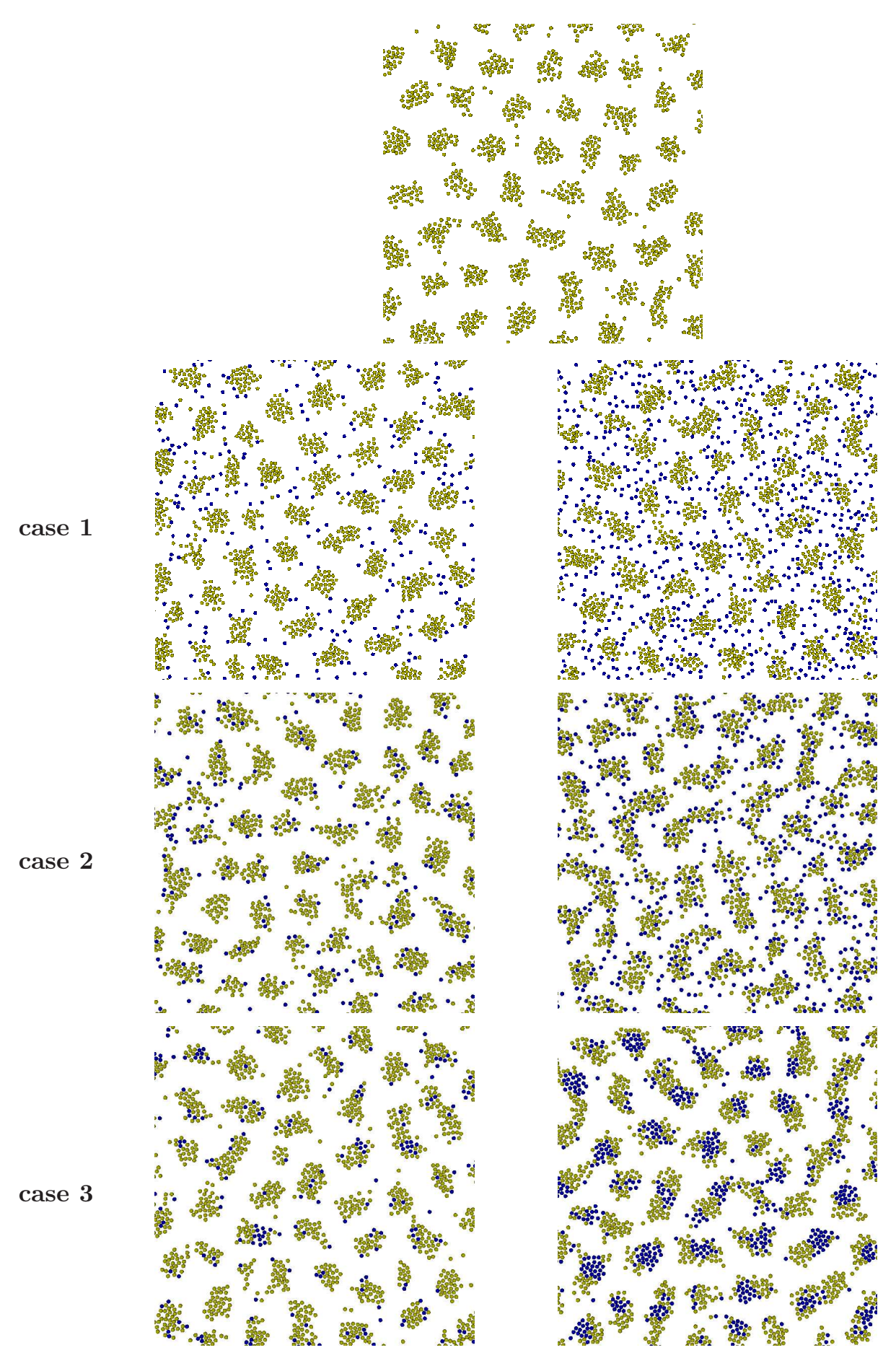

Figure 2. (color online) Top panel: snapshot of an equilibrated fluid at $\rho_{\mathrm{f}}=0.2$. Other panels: selected, representative snapshots of systems located in the $\left(\rho_{\mathrm{f}}, \rho_{\mathrm{m}}\right)$-plane along path $A$, specified in figure 1 The three rows correspond to cases 1 to 3 , as specified in the text, the two columns correspond to a matrix density $\rho_{\mathrm{m}}=0.035$ (left column) and $\rho_{\mathrm{m}}=0.1$ (right column), respectively. Light (yellow) particles - fluid particles, dark (blue) particles - matrix particles. 


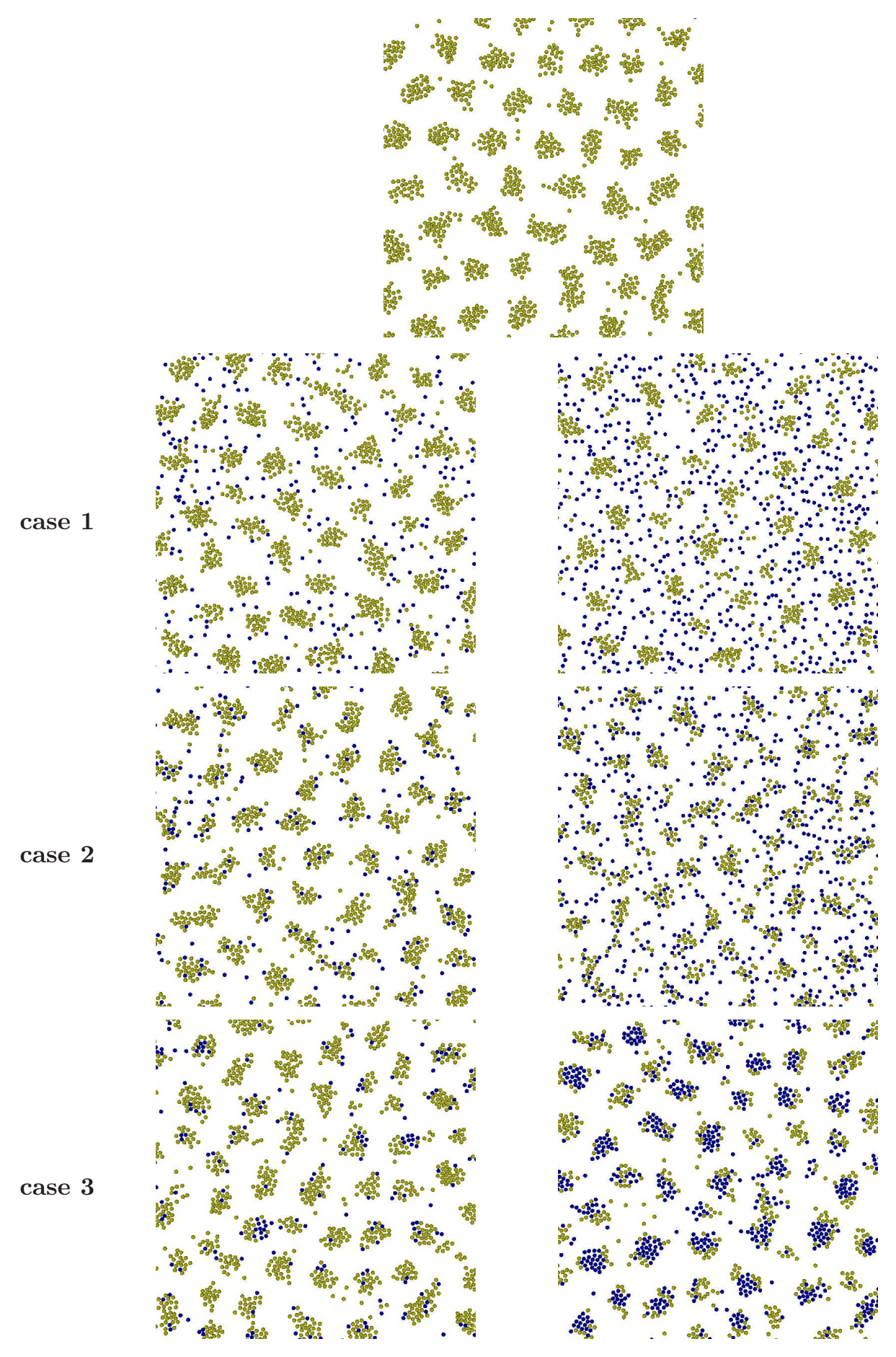

Figure 3. (color online) Top panel: snapshot of an equilibrated fluid at $\rho_{\mathrm{f}}=0.2$. Other panels: selected, representative snapshots of systems located in the $\left(\rho_{\mathrm{f}}, \rho_{\mathrm{m}}\right)$-plane along path $B$, specified in figure 1. The three rows correspond to cases 1 to 3 , as specified in the text, the two columns correspond to a matrix density $\rho_{\mathrm{m}}=0.035$, i.e., $\rho_{\mathrm{f}}=0.165$ (left column) and $\rho_{\mathrm{m}}=0.1$, i.e., $\rho_{\mathrm{f}}=0.1$ (right column), respectively. Light (yellow) particles - fluid particles, dark (blue) particles - matrix particles. 
a typical equilibrium configuration of the fluid (i.e., $\rho_{\mathrm{m}}=0$ ) is depicted. We start the discussion of the snapshots with three panels of the left column where we consider a matrix at density $\rho_{\mathrm{m}}=0.035$. As we now immerse the fluid into the quenched matrix configuration we observe only a weak effect of the immobile matrix particles on the formation of clusters by the fluid particles: from a qualitative visual inspection of the snapshots we learn that the number of clusters as well as their size is essentially the same for three different interaction scenarios considered in cases 1 to 3 . We only notice that for case 1 , the essentially inert matrix particles are located predominantly outside the clusters, while in case 3 , i.e. when both types of particles interact via IR potentials, the matrix particles have become part of the clusters. This means that in this case the matrix particles act as nucleation centers for the clusters formed by the fluid particles. We now repeat this experiment at a higher matrix density, i.e. at $\rho_{\mathrm{m}}=0.1$. Now the matrix particles do have a distinct effect on the cluster formation of the fluid particles. In case 1 the rather compact (i.e., close-tospheric) clusters of the fluid particles are formed nearly exclusively in those regions which have been left void by the matrix particles. As we proceed to case 2, i.e. as we switch on an IR-tail in the matrix-fluid particle interaction, the situation changes drastically: due to a rather high matrix density, the clusters are forced to arrange predominantly 'around' the matrix particles and with respect to case 1 , the clusters have grown considerably in size and tend to have elongated shapes. Finally, in case 3 where all interactions are of the IR type, the matrix particles are fully included in the clusters formed by the fluid particles; in addition, the clusters have increased considerably in size compared to the pure fluid (cf. top panel of figure 21).

We now perform a similar inspection of simulation snapshots for systems located in the $\left(\rho_{\mathrm{f}}, \rho_{\mathrm{m}}\right)$ plane along path $B$ specified in figure 1 results are depicted in figure 3. Now the total density, $\rho_{\mathrm{t}}=\rho_{\mathrm{f}}+\rho_{\mathrm{m}}$, is kept fixed and we increase $\rho_{\mathrm{m}}$ from 0 to 0.1 , i.e., we decrease $\rho_{\mathrm{f}}$ from 0.2 to 0.1 . Again, along this path $\rho_{\mathrm{m}} \leqslant 0.1$, i.e. the matrix particles are not capable of forming clusters at this temperature. Similar to the case of systems located along path $A$, we start in the top panel of figure 3 with a typical equilibrium configuration of the fluid (i.e., $\rho_{\mathrm{m}}=0$ and hence $\rho_{\mathrm{f}}=0.2$ ). For the snapshots of the left column we have chosen $\rho_{\mathrm{m}}=0.035$ and hence $\rho_{\mathrm{f}}=0.165$. As we immerse the fluid particles into the matrix formed by HS particles (case 1), we observe that the clusters formed by the fluid particles populate preferentially the space left void by the matrix particles, including in a few cases the essentially inert matrix particles, if imposed by space requirements. As we now turn on the IR-tail first in the fluid-matrix and then in the matrix-matrix interactions, the matrix particles become nucleation centers of the emerging clusters formed by the fluid particles. In particular in case 3, where the matrix particles also interact via an IR interaction, the clusters are formed both by the fluid and the matrix particles, i.e., we observe a microphase which essentially corresponds to the pure fluid case (see top panel). As we repeat a similar gedanken-experiment at $\rho_{\mathrm{m}}=0.1$ (and hence $\rho_{\mathrm{f}}=0.1$ ), we observe the following microphase formation scenario: in case 1 , where the matrix particles are simple HS, the fluid particles do form clusters; at first sight this is quite astonishing, since - according to the phase diagram depicted in figure 4 of [17] - at a fluid of density $\rho_{\mathrm{f}}=0.1$ and at the temperature considered in this contribution, a pronounced cluster formation is rather unlikely. However, the rather elevated matrix density essentially reduces the available space so that the effective fluid density is larger than the nominal fluid density $\rho_{\mathrm{f}}=0.1$, forcing thereby the fluid particles to form clusters. As we proceed to case 2, i.e., as we switch on the IR-tail in the fluid-matrix interaction, microphase formation is strongly suppressed: obviously, under the effect of the external potential exerted by the matrix particles, the fluid particles are not capable of forming clusters. Finally, in case 3 we return to a pronounced cluster-microphase formation where the matrix particles represent nucleation centers for the fluid particles.

\subsection{Static structure factors}

We now examine our data on a more quantitative level and start to discuss the results obtained for the static structure factor, $S_{\mathrm{ff}}(k)$, providing information on the correlations between the fluid 
particles. $S_{\mathrm{ff}}(k)$ has been calculated during the simulation run according to the following expression

$$
S_{\mathrm{ff}}(k)=\frac{1}{N_{\mathrm{f}}} \overline{\left\langle\rho_{\mathrm{f}}(\mathbf{k}) \rho_{\mathrm{f}}(-\mathbf{k})\right\rangle} \quad \text { with } \quad \rho_{\mathrm{f}}(\mathbf{k})=\sum_{j=1}^{N_{\mathrm{f}}} \exp \left(\mathrm{ik} \cdot \mathbf{r}_{j}\right) .
$$

The $\mathbf{r}_{j}$ are the positions of the fluid particles and the vectors $\mathbf{k}$ are compatible with the square geometry of the simulation box (see, e.g. [20]). As a consequence, in the low- $k$ regime the number of $\mathbf{k}$-vectors available is smaller, the structure factors are not as smooth as for larger $k$-values. Here $\langle\cdots\rangle$ denotes the thermal average over the degrees of freedom of the fluid particles at a given matrix configuration while $\cdots$ stands for the average over different but equivalent matrix configurations.

case 1

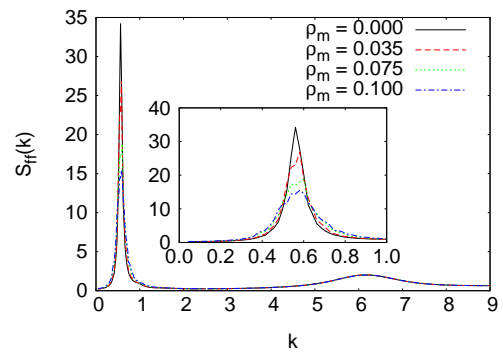

case 2
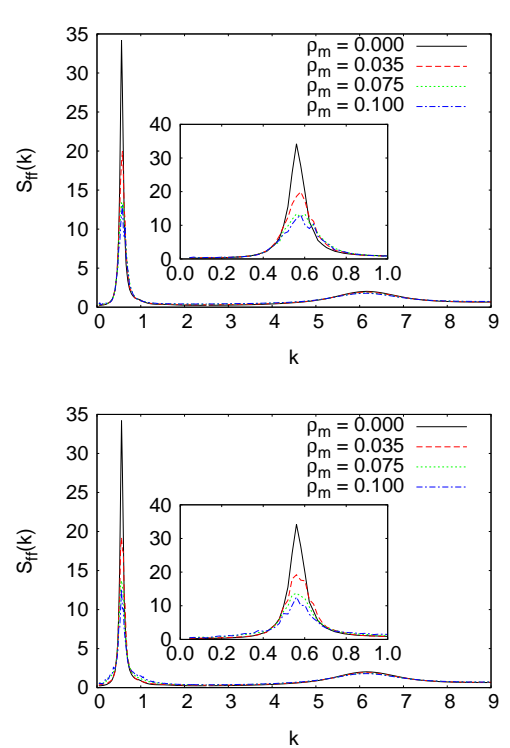
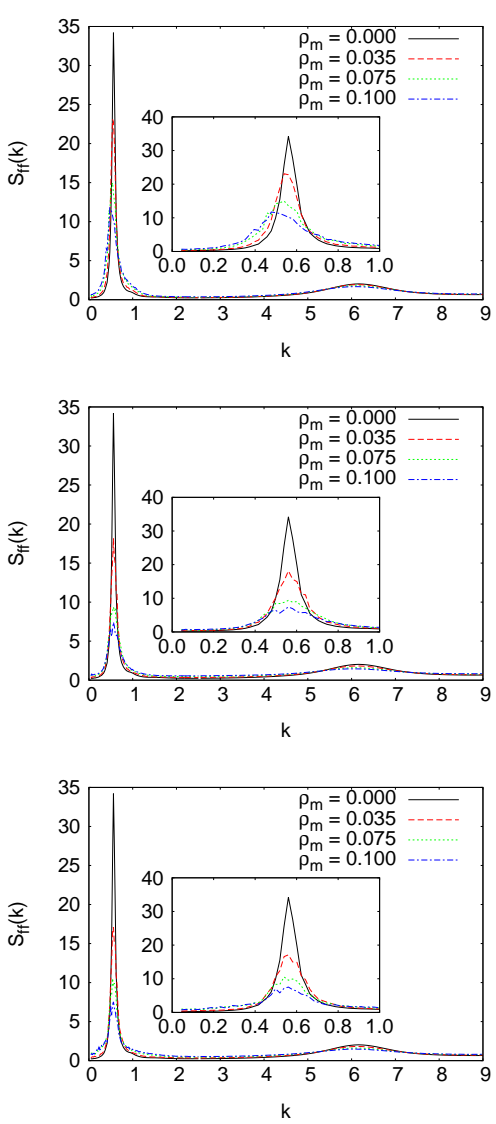

Figure 4. Fluid-fluid static structure factor, $S_{\mathrm{ff}}(k)$ as a function of $k$ for systems located in the $\left(\rho_{\mathrm{f}}, \rho_{\mathrm{m}}\right)$-plane along path $A$ (left column) and along path $B$ (right column), as specified in figure 1 The three rows correspond to cases 1 to 3 , as specified in the text. Different line symbols correspond to different values of $\rho_{\mathrm{m}}$ as labeled. The insets show enlarged views of the low- $k$ range.

Results for $S_{\mathrm{ff}}(k)$ are depicted in figure 4, in the left (right) column we display the static structure factor for systems located in the $\left(\rho_{\mathrm{f}}, \rho_{\mathrm{m}}\right)$-plane along path $A$ (path $B$ ), respectively, as defined in figure 1, considering the three different sets of interactions specified in section 2 For reference, the static structure factor for the pure fluid $\left(\rho_{\mathrm{f}}=0.2\right.$ and $\left.\rho_{\mathrm{m}}=0\right)$ is shown in all panels.

All structure factors display the same two characteristic features: they have a rather small peak at $k \sim 6.2$, denoting the interparticle correlations. They show a pronounced first peak at $k \sim 0.6$, corresponding to the intercluster correlations. These two $k$-values provide some rough estimate that the average interparticle distances are by a factor of ten smaller than the average 
intercluster distances. As we increase the matrix density $\rho_{\mathrm{m}}$, we observe throughout a distinct decrease in the height of the first peak, while the $\rho_{\mathrm{m}}$-dependence of the height of the second peak is rather weak. With respect to the different interaction scenarios (i.e., cases 1 to 3 ) we observe distinct differences in how the height of the first peak decreases as $\rho_{\mathrm{m}}$ is increased: in case 1 , where the matrix particles are essentially inert hard sphere particles, the decay in the peak height is rather slow with increasing $\rho_{\mathrm{m}}$ and thus even at the highest $\rho_{\mathrm{m}}$-value investigated, the intercluster correlations are still quite pronounced. As we turn on the tail in the matrix-matrix interactions (case 2) and, finally, in the fluid-matrix interactions (case 3), the height of the first peak decreases much faster as $\rho_{\mathrm{m}}$ increases, reflecting a rapid decrease in the correlations between clusters due to the presence of matrix particles. As we now proceed to systems located in the $\left(\rho_{\mathrm{f}}, \rho_{\mathrm{m}}\right)$-plane along path $B$, we observe the following scenario. The tendencies in the decay of the first peak with increasing $\rho_{\mathrm{m}}$ (and, consequently, with decreasing $\rho_{\mathrm{f}}$ ), while we also proceed from case 1 to 3 , are similar to the ones reported for path $A$. Furthermore we observe that only for case 1 the positions of the first peak shift to lower $k$-values, as $\rho_{\mathrm{m}}$ increases, indicating an increase in the intercluster distance. We interpret these observations as follows: in case 1 (pure HS matrix), the fluid particles, interacting via IR potentials, can form well-defined clusters at low $\rho_{\mathrm{m}}$-values since the matrix particles do not represent an essential spatial restriction (cf. corresponding snapshots in figure 3). However, at an increased matrix density, less space is left available for the fluid particles to form clusters, which are now forced to emerge in the reduced space left free by the matrix particles: clusters are now considerably more dispersed than for small $\rho_{\mathrm{m}}$-values and are smaller

case 1
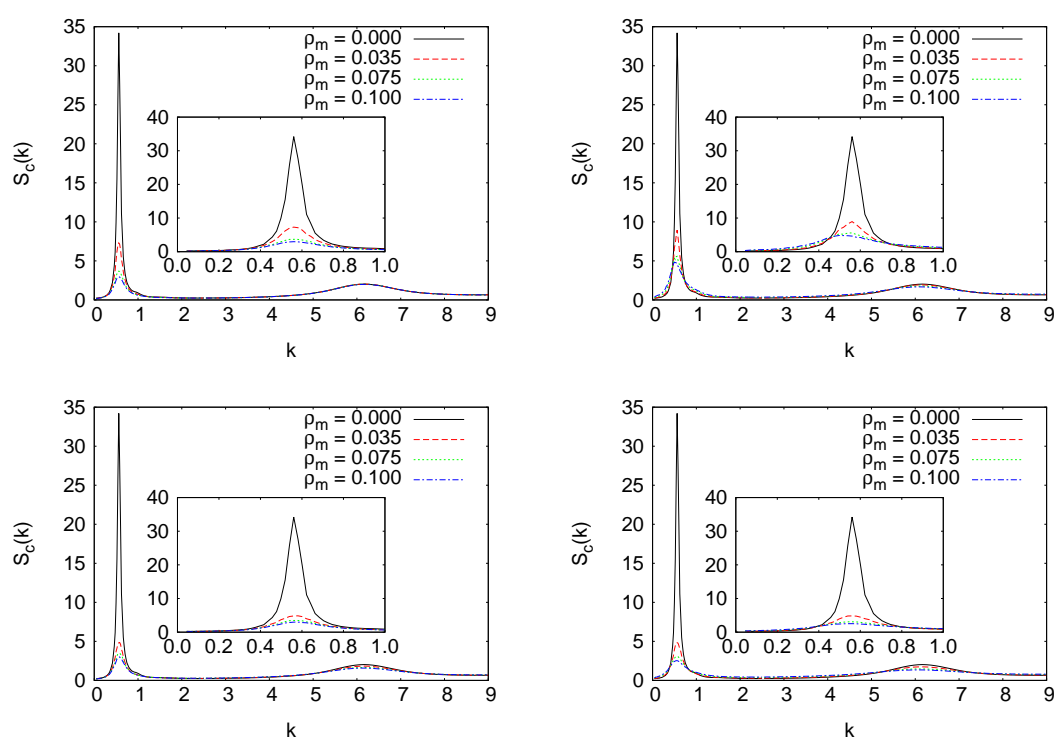

case 2
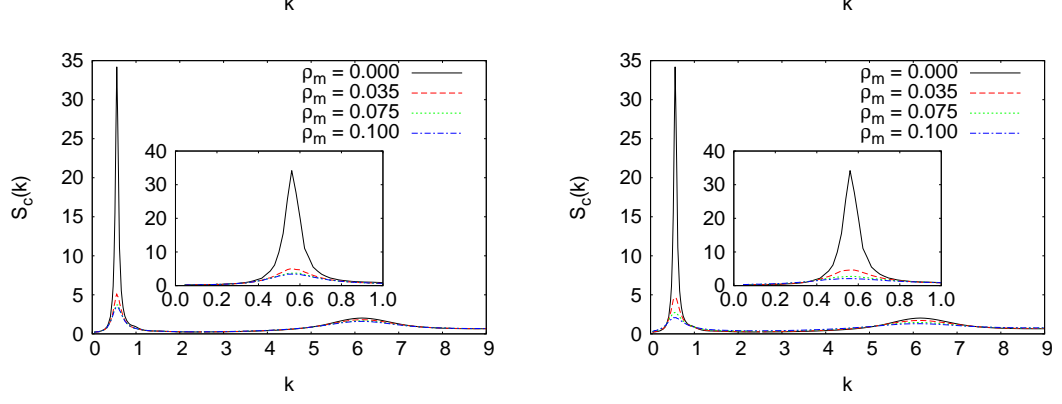

Figure 5. Connected structure factor, $S_{\mathrm{c}}(k)$ as a function of $k$ for systems located in the $\left(\rho_{\mathrm{f}}, \rho_{\mathrm{m}}\right)$ plane along path $A$ (left column) and along path $B$ (right column), as specified in figure 1 The three rows correspond to cases 1 to 3 , as specified in the text. Different line symbols correspond to different values of $\rho_{\mathrm{m}}$ as labeled. The insets show enlarged views of the low- $k$ range. 
in size (again, cf. the corresponding snapshots in figure 2). The situation is different for cases 2 and 3 as an IR-tail is switched on in the fluid-matrix and/or in the matrix-matrix interactions: the attractive components in these interaction tails foster the formation of a cluster of the fluid particles. This is possible in case 2 despite the presence of the matrix particles even at higher $\rho_{\mathrm{m}}$-values while in case 3 the matrix particles are nucleation centers of the clusters formed by the fluid particles. Thus, in both cases the intercluster distance remains essentially unchanged upon increasing $\rho_{\mathrm{m}}$, and consequently the position of the first peak does not change with $\rho_{\mathrm{m}}$ increasing.

We have also evaluated the connected structure factor, $S_{\mathrm{c}}(k)$, from the particle positions defined via

$$
S_{\mathrm{c}}(k)=\frac{1}{N_{\mathrm{f}}} \overline{\left\langle\delta \rho_{\mathrm{f}}(\mathbf{k}) \delta \rho_{\mathrm{f}}(-\mathbf{k})\right\rangle},
$$

where $\delta \rho_{\mathrm{f}}(\mathbf{k})=\rho_{\mathrm{f}}(\mathbf{k})-\left\langle\rho_{\mathrm{f}}(\mathbf{k})\right\rangle$. This structure factor provides information about the correlations between the fluid particles that are not mediated via the matrix particles. Results are shown in figure 5] in the left (right) column we display the static structure factor for systems located in the $\left(\rho_{\mathrm{f}}, \rho_{\mathrm{m}}\right)$-plane along path $A$ (path $B$ ), respectively, as defined in figure 1, considering three different sets of interactions specified in section 2. For reference, the static structure factor for the pure fluid $\left(\rho_{\mathrm{f}}=0.2\right)$, to which $S_{\mathrm{c}}(k)$ reduces for $\rho_{\mathrm{m}}=0$, is shown in all panels.

While the variation of the height of the second peak in $S_{\mathrm{c}}(k)$ at $k \sim 6.2$ with the densities and the types of interactions shows the same behaviour as the one reported for $S_{\mathrm{ff}}(k)$, we do observe a distinctively different behaviour on the variation of the height of the first peak at $k \sim 0.6$ as the system parameters are changed. Throughout we observe a more pronounced decrease in the height of this peak as $\rho_{\mathrm{m}}$ increases, corresponding to a gradually increasing suppression of direct correlations between the fluid particles: at $\rho_{\mathrm{m}}=0.1, S_{\mathrm{c}}(k \sim 0.6) \sim 3( \pm 1)$ while $S_{\mathrm{ff}}(k \sim 0.6) \sim 12( \pm 3)$. In addition, the particular shape of the fluid-matrix interaction has also an effect on the decrease of the height of the first peak of the structure factor: the essentially inert HS matrix particles (case 1) allow for a reasonable amount of direct correlations between the particles while in case 3 , where both fluid and matrix particles interact via the same type of potential, the correlations are strongly suppressed.

\subsection{Mean square displacement}

Finally, in figure 6 we display the mean square displacement

$$
\left\langle\delta r^{2}(t)\right\rangle=\langle|\mathbf{r}(t)-\mathbf{r}(0)|\rangle
$$

obtained from the positions of the particles over a respective range in time, measured in terms of Monte Carlo sweeps where one sweep corresponds to $N_{\mathrm{f}}$ attempted displacement moves (see, e.g., [21]). For systems located in the $\left(\rho_{\mathrm{f}}, \rho_{\mathrm{m}}\right)$-plane along path $A$ (cf. figure 1), we observe that an increase in the matrix density $\rho_{\mathrm{m}}$ leads in all cases to a decrease in the diffusion constant $D$ which represents the slope of the essentially linear mean square displacement curves. The results indicate that this decrease in $D$ is more rapid with $\rho_{\mathrm{m}}$ increasing in case 1 than in the other two cases; in particular in case 3 the decrease in $D$ is relatively small as $\rho$ increases. We think that these observations are the result of two competing effects: on the one hand the fluid particles are certainly more trapped within the clusters in cases 2 and 3, while in case 1 the HS matrix particles form inert obstacles. On the other hand, the neatly defined clusters in case 2 and, in particular, in case 3 (cf. corresponding snapshots in figure 2) offer large void regions of space where the fluid particles can propagate more freely than in the corresponding case of a pure HS matrix (cf. corresponding snapshots in figure 2). For the respective cases, the complex interplay of these two effects leads to the above mentioned decrease in $D$ as $\rho_{\mathrm{m}}$ increases. For systems located in the $\left(\rho_{\mathrm{f}}, \rho_{\mathrm{m}}\right)$-plane along path $B$, an increasing matrix density $\rho_{\mathrm{m}}$ leads to an increase in the diffusion constant. This increase is a bit more pronounced in cases 1 and 2 than in case 3 . We point out that in case 1 a distinct non-monotonous behaviour of the diffusion constant at small $\rho_{\mathrm{m}}$-values $\left(\rho_{\mathrm{m}} \lesssim 0.035\right)$ is observed as the matrix density increases. A similar behaviour is encountered in cases 2 and 3 only for matrix densities up to $\rho_{\mathrm{m}} \simeq 0.01$. 

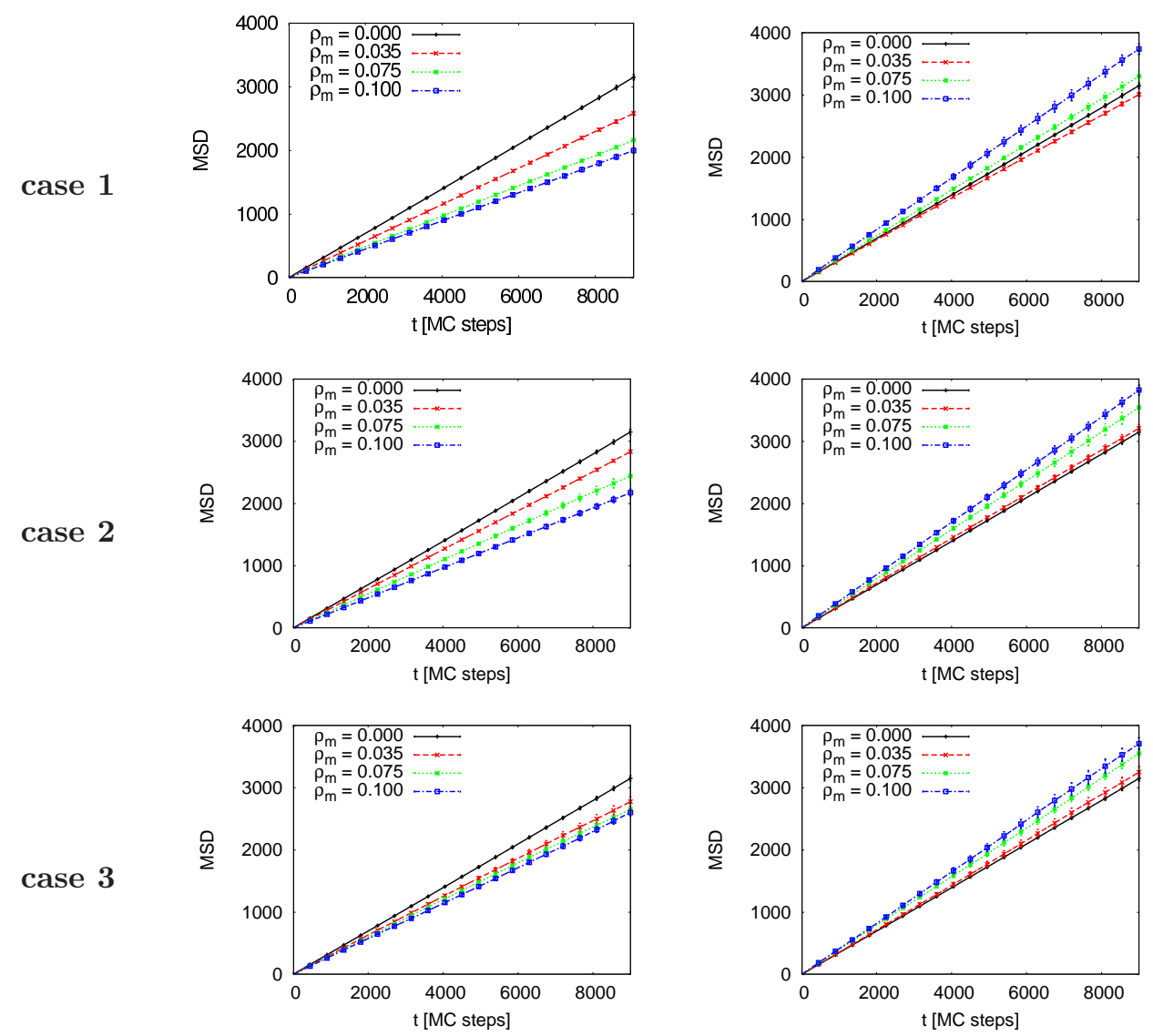

Figure 6. Mean square displacement, $\left\langle\delta r^{2}(t)\right\rangle$, as a function of time (measured in terms of Monte Carlo steps, where one step corresponds to 100 Monte Carlo sweeps) for systems located in the $\left(\rho_{\mathrm{f}}, \rho_{\mathrm{m}}\right)$-plane along path $A$ (left column) and along path $B$ (right column), as specified in figure 1. The three rows correspond to cases 1 to 3 , as specified in the text. Different line symbols correspond to different values of $\rho_{\mathrm{m}}$ as labeled.

\section{Conclusions}

In this contribution we have investigated the cluster microphase formation of a two-dimensional fluid annealed in the presence of a quenched matrix configuration of fluid particles. In all cases investigated the fluid particles interact via a hard sphere potential with an adjacent tail of competing interactions. In an effort to study the cluster formation of the fluid particles under the effect of the matrix particles we have varied both the fluid and the matrix densities, $\rho_{\mathrm{f}}$ and $\rho_{\mathrm{m}}$, as well as the fluid-matrix and the matrix-matrix interactions, ranging from simple hard spheres to hard spheres with competing tail interactions. Our investigations are based on extensive Monte Carlo simulations. The observables, such as static and dynamic correlation functions, are obtained by a double averaging procedure: one average is taken over the degrees of freedom of the mobile fluid particles for a particular matrix realization, the other one is taken over different but equivalent matrix configurations.

We have discussed the observed phenomena on a qualitative level by visual inspection of the selected representative snapshots and on a quantitative level by evaluating the static structure [i.e., the structure factors $S_{\mathrm{ff}}(k)$ and $\left.S_{\mathrm{c}}(k)\right]$ as well as the mean square displacement, $\left\langle\delta r^{2}(t)\right\rangle$. These results provide clear evidence that the matrix does have a distinct effect on the microphase formation, which can be summarized as follows: small matrix densities leave the cluster formation essentially unaffected while larger $\rho_{\mathrm{m}}$-values do effect the microphase formation. Here the explicit 
shape of the fluid-matrix and of the matrix-matrix interactions become relevant: simple hard sphere matrix particles can effect the cluster formation by the fact that they are capable of reducing the space available to the fluid particles, increasing thereby the effective matrix density; on the other hand, if the interactions between the fluid and the matrix particles is of the same type, we have found that the matrix particles act as nucleation centers for the emerging cluster microphase of the fluid particles.

\section{Acknowledgements}

Financial support by the Austrian Research Fund (FWF) under Proj. Nos. P19890-N16 and W004 is gratefully acknowledged. The authors are indebted to Daniele Coslovich (Montpellier) and Jan Kurzidim (Wien) for helpful discussions.

One of us (GK) is in particular grateful to Yura Kalyuzhnyi to whom this contribution is dedicated: our close cooperation has been extremely fruitful, both from the scientific and from the personal point of view.

\section{References}

1. Klokkenburg M., Dullens R.P.A., Kegel W.K., Erné B.H., Philipse A.P., Phys. Rev. Lett., 2006, 96, 037203; doi 10.1103/PhysRevLett.96.037203

2. Heath J.R., Gelbart W.M., Sear R.P., Chaney S., Faraday Discuss., 1999, 112, 299; doi $10.1039 / \mathrm{a} 809582 \mathrm{e}$

3. Elias F., Flament C., Bacri J.C., Neveu S., J. Phys. I, 1997, 7, 711; doi:10.1051/jp1:1997186

4. Ghezzi F., Earnshaw J.C., J. Phys. Condens. Matter, 1997, 9, L517; doi 10.1088/0953-8984/9/37/004.

5. Seul M., Andelman D. Science, 1995, 267, 476; doi 10.1126/science.267.5197.476

6. Imperio A., Reatto L., J. Phys. Condens. Matter, 2004, 16, S3769; doi:10.1088/0953-8984/16/38/001.

7. Imperio A., Reatto L., J. Chem. Phys., 2006, 124, 164712; doi 10.1063/1.2185618

8. Pini D., Parola A., Reatto L., J. Phys. Condens. Matter, 2006, 18, S2305; doi: $10.1088 / 0953-8984 / 18 / 36 /$ S06

9. Imperio A., Reatto L., Phys. Rev. E, 2007, 76, 040402; doi 10.1103/PhysRevE.76.040402

10. Imperio A., Reatto L., Zapperi S., Phys. Rev. E, 2008, 78, 021402; doi:10.1103/PhysRevE.78.021402.

11. Archer A.J., Phys. Rev. E, 2008, 78, 031402; doi 10.1103/PhysRevE.78.031402

12. Imperio A., Pini D., Reatto R. - In: Proc. Workshop "Collective Phenomena in Macroscopic Systems", eds.: Bertin G., Pozzoli R., Rome M., Sreenivasan K.R. World Scientific, Singapore, 2007.

13. Archer A.J., Wilding N.B., Phys. Rev. E, 2007, 76, 031501; doi 10.1103/PhysRevE.76.031501

14. Archer A.J., Pini D., Evans R., Reatto L., J. Chem. Phys., 2007, 126, 014104; doi 10.1063/1.2405355.

15. Archer A.J., Ionescu C., Pini D., Reatto L., J. Phys. Condens. Matter, 2008, 20, 415106; doi:10.1088/0953-8984/20/41/415106.

16. Lee L.L., Hara M.C., Simon S.J., Ramos F.S., Winkle A.J., Bomont J.M., J. Chem. Phys., 2010, 132, 074505; doi $10.1063 / 1.3308648$

17. Schwanzer D.F., Kahl G., J. Phys. Condens. Matter, 2010, 22, 415103; doi: 10.1088/0953-8984/22/41/415103.

18. Kurzidim J., Coslovich D., Kahl G., Phys. Rev. Lett., 2009, 103, 138303; doi:10.1103/PhysRevLett.103.138303.

19. Kurzidim J., Coslovich D., Kahl G., Phys. Rev. E, 2010, 82, 041505; doi 10.1103/PhysRevE.82.041505.

20. Schwanzer D. F., Coslovich D., Kurzidim J., Kahl G., Mol. Phys., 2009, 107, 433; doi $10.1080 / 00268970902845321$

21. Coslovich D., Strauss L, Kahl G., Soft Matter, 2011, 7, 2127; doi 10.1039/c0sm00545b 


\title{
Двовимірні системи із конкуруючими взаємодіями: формування мікрофази під впливом невпорядкованого пористого середовища
}

\author{
Д.Ф. Шванцер, Г. Каль
}

Інститут теоретичної фізики та центр чисельного матеріалознавства, Технічний університет Відня, Відень, Австрія

\begin{abstract}
Нами досліджено вплив невпорядкованого пористого середовища на утворення кластерної мікрофази у двовимірній системі, у якій мають місце конкуруючі взаємодії між частинками. 3 цією метою ми здійснили грунтовне моделювання методом Монте-Карло, систематично змінюючи густину плину та середовища, а також взаємодію між частинками середовища і взаємодію між частинками середовища та плину. Наші результати доводять, що середовище істотно впливає на формування мікрофази частинок плину: якщо частинки взаємодіють як між собою так і з частинками плину через звичайний потенціал твердих сфер, то вони, по суті, зменшують доступний об'єм, у якому частинки плину формують кластерну мікрофазу. 3 іншого боку, якщо розглядати далекосяжну частину взаємодій “середовище - середовище"і “середовище - плин”, то частинки середовища стають центрами нуклеації для кластерів, утворених частинками плину.
\end{abstract}

Ключові слова: м'яка речовина, пористі середовища, утворення мікрофази, статична структура, динамічні властивості 